\title{
Bubble enhanced heat transfer from a vertical heated surface
}

\author{
Brian Donnelly $^{\mathrm{a}, *}$, Darina B. Murray ${ }^{\mathrm{a}} \&$ Tadhg S. O'Donovan ${ }^{\mathrm{b}}$ \\ ${ }^{a}$ Department of Mechanical \& Manufacturing Engineering, \\ Trinity College Dublin, Ireland \\ ${ }^{b}$ School of Engineering \& Physical Sciences, Heriot-Watt University, \\ Edinburgh, United Kingdom
}

\begin{abstract}
A rising bubble in a liquid can greatly enhance heat transfer from heated surfaces by acting like a bluff body, displacing fluid as it moves and via the wake generated by the bubble, increasing the mixing of the liquid. The current research quantifies the effect a single free rising ellipsoidal air bubble has on heat transfer from a vertical heated block immersed in water. By measuring the time varying heat transfer and tracking the bubble dynamics, further understanding of the heat transfer mechanism is achieved. Both the plane in which the bubble oscillates and the proximity of the bubble path are shown to influence the surface heat transfer.
\end{abstract}

Keywords: Bubble, Heat Transfer, Buoyancy Driven

\footnotetext{
*Corresponding Author. Tel.: +353-1-896-3920; Fax.: +353-1-679-5554

E-mail address: donnelbg@tcd.ie (Brian Donnelly)
} 


\section{Introduction}

Bubbly flows occur in many engineering applications such as steam generation, electronic cooling systems and chemical reactors. These bubbles can be present either due to phase change or by addition of a gas to the flow. An important aspect of bubble dynamics is the interaction between a rising bubble and a vertical heated surface. It is known that this can increase heat transfer from the surface and is an effective and efficient method for doing so. In an earlier study related to the present investigation, Delauré et al. [2003] investigated heat transfer enhancement from a vertical heated block due to a single rising ellipsoidal air bubble in water. Digital Particle Image Velocimetry (DPIV) was performed in order to explore the mechanism of wake generation and interaction with the heated surface. From this, velocity vector plots clearly illustrated the generation of so called "horse shoe" vortices responsible for increased mass flow in the vicinity of the heated surface. Two enhancement mechanisms were identified; first the heat transfer coefficient was found to respond closely to changes in flow velocity, with the shedding of vortices interacting with the thermal boundary layer in a positive or a negative manner. Secondly, the zigzagging motion of the bubble draws cooler water in from the surroundings which impacts on the block surface. It was found that a combination of the two mechanisms results in the largest heat flux enhancement.

Another area of importance in bubble enhanced heat transfer is the sliding bubble phenomenon, when a rising bubble impacts on a downward facing heated surface and slides along its length. In a study by Manickam and Dhir [2003], holographic interferometry was used to visualise the variation in fluid temperature surrounding a sliding vapour bubble. The heat transfer to and from the bubble was quantified. It is known that a liquid layer exists between the bubble and the heated surface but its thickness and contribution to heat transfer is much debated. They concluded that the vapour bubble continues to grow as it slides along the heated surface and provided a power law equation to calculate the growth rate, which is 
achieved by evaporation of the thin liquid layer. Condensation between the bubble and bulk fluid can also occur (effectively reducing the volume) but this is out weighed generally by evaporation. An earlier study performed by Qiu and Dhir [2002] into sliding bubbles illustrates the existence of a wedge-like liquid gap in front of the bubble determined by the angle made with the surface. The apparent wedge angle is seen to increase as the heater inclination angle increases and the wedge length increases with bubble size. In magnified photography the wedge is seen to almost penetrate to the down stream side of the bubble, thus it is connected to the thin liquid film. The relative contribution of the liquid layer evaporation to the total heat transfer rate was found to be small in their work. It was observed for plate angles above $60^{\circ}$ that the bubble began to lift off and then hop along the surface, this was found to be due to a recoil force provided by evaporation and a shear lift force on the bubble. Cornwell and Grant [1998] also report the existence of a thin evaporating liquid layer beneath a bubble sliding under a horizontal tube. Both water and Flutec (a refrigerant) were used in the study. High speed photography and thermochromic liquid crystal paints were used to evaluate the contribution of the evaporation of this layer to the overall heat transfer. Results indicated that evaporation of the liquid layer can cause "dry out” (a dry spot between the bubble and surface) which is quenched by the surrounding fluid after the bubble moves away. They concluded that neither the bubble motion nor the liquid layer evaporation is dominant under all conditions. They also noted bubbles that pass close to the surface may receive heat from the outer reaches of the boundary layer, increasing heat transfer. The existence of "dry out” was also found by Yan et al. [1997] to lead to local hot spots on the heated surface. Sliding and sticking bubbles were observed under inclined planes and curved surfaces with heat transfer enhancement factors of 3 to 5 reported close to the trailing edge of the bubble compared to the undisturbed state. For large, slow moving bubbles, evaporation was found to be the dominant heat transfer mechanism, whereas for smaller bubbles the 
reduction in evaporation was compensated by the higher velocity and therefore higher wake turbulence. In a study by Atmane and Murray [2001] on bubble dynamics in nucleate boiling around a cylinder, heat flux enhancement of up to 7 times was recorded using a similar technique to the present study, with the maximum occurring when the bubble was directly above the sensor. It was found that heat transfer was affected by both bubble detachment and by the wake generated by the bubble, evidenced by the long enhancement times. Thorncroft and Klausner [1999] conclude that sliding bubbles can account for as much as $52 \%$ of the total energy transfer, outweighing the contribution of bubble nucleation.

In the study of Qiu and Dhir [2002], holographic interferometry is used to visualise both the near and far wake of the bubble for angles of plate inclination of $15^{\circ}$ and $75^{\circ}$ from the horizontal. At $15^{\circ}$ the thermal boundary layer is seen to expand as the liquid tries to flow around the bubble. Vortices were observed to form downstream of the bubble, detach, and move into the bulk fluid where they dissipated. This results in an increase in heat transfer as heated fluid is moved away from the surface and cooler fluid replaces it. For plate angles of $75^{\circ}$ the thermal boundary layer was no longer laminar and even without the bubble vortices were observed to roll over the boundary layer. Thus, the scope for heat transfer enhancement by small bubbles at $75^{\circ}$ inclination was limited.

In a study by Brücker [1999] on the wake of bubbles and the wake influence on bubblebubble interactions, DPIV and high speed photography were used to obtain the temporal evolution of the flow field in the near wake of bubbles of 5 to $7 \mathrm{~mm}$ diameter rising in water. These bubbles were found to zigzag at a frequency of approximately $4.2 \mathrm{~Hz}$ with a maximum lateral velocity of about $100 \mathrm{~mm} / \mathrm{s}$. Bubbles followed a sinusoidal path with a slight drift attributed to a net lift on one side of the bubble. Shape fluctuations were observed for bubbles of this size and were attributed to an uneven pressure distribution in the equatorial plane at the inversion points of its motion. The existence of hairpin vortices was confirmed by DPIV 
performed in a plane perpendicular to the bubble flow direction. This showed the alternate generation of a pair of counter-rotating vortices close to the bubble base.

By using the hydrogen bubble tracer technique, Bhaga and Weber [1981] visualised both a steady wake with a standing eddy and open unsteady wakes behind bubbles rising in liquids of different viscosity. A shape regime map was also provided and shown to depend on the Morton, Weber and Reynolds numbers. This map allows us to predict the expected shape of the bubble i.e. spherical, ellipsoidal, cap etc.

The work of Ellingsen and Risso [2001] confirmed the sinusoidal motion of the bubble and the drift described by Brücker [1999] using smaller bubbles (approximately 2.5mm). A second oscillation, of smaller amplitude, was noted but this time in a direction parallel to the bubble flow direction. They deemed the effect of surfactants on bubble motion to be negligible but this finding was for smaller bubbles than investigated in the present study. A standing eddy behind the bubble, as seen by Bhaga and Weber [1981], was also reported. It was suggested that it is the wake which causes bubble path oscillations. Saffman [1956] theorised that the observed zigzagging of a bubble was caused by the interaction of an oscillating wake and the instability of the motion near the front of the bubble. An oscillation frequency of $7 \mathrm{~Hz}$ was found for air bubbles in water and it was suggested that this is independent of bubble size.

The present research aims to quantify the interaction between a single rising ellipsoidal bubble and a heated vertical plate. Based on this review it is clear that, from a heat transfer perspective, bubbles sliding under inclined, curved and planar surfaces have been investigated more extensively than bubbles rising past a vertical surface. The present experimental study is aimed at providing simultaneous, synchronised information on bubble motion and surface heat flux for rising bubbles of $4 \mathrm{~mm}$ diameter. The effects of bubble proximity to the plate and bubble oscillation plane on heat transfer from the surface were 
investigated using high speed imagery synchronised with a hot film heat flux sensor, providing simultaneous time dependent bubble position and heat flux data.

\section{Experimental Rig}

The experimental apparatus facilitates the simultaneous measurement of heat flux and temperatures and the recording of bubble motion. It comprises four main components, the water tank, the bubble injection system, the instrumented test surface and the imaging system, as shown in figure 1.

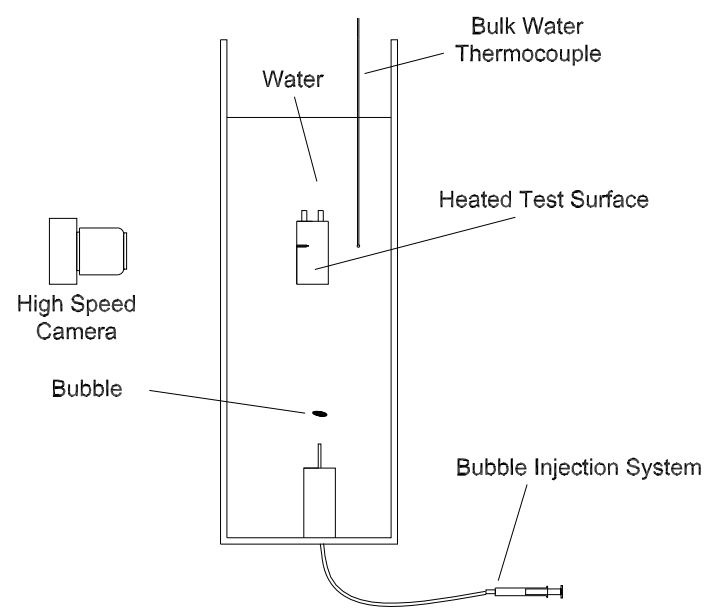

(a) Test Water Tank

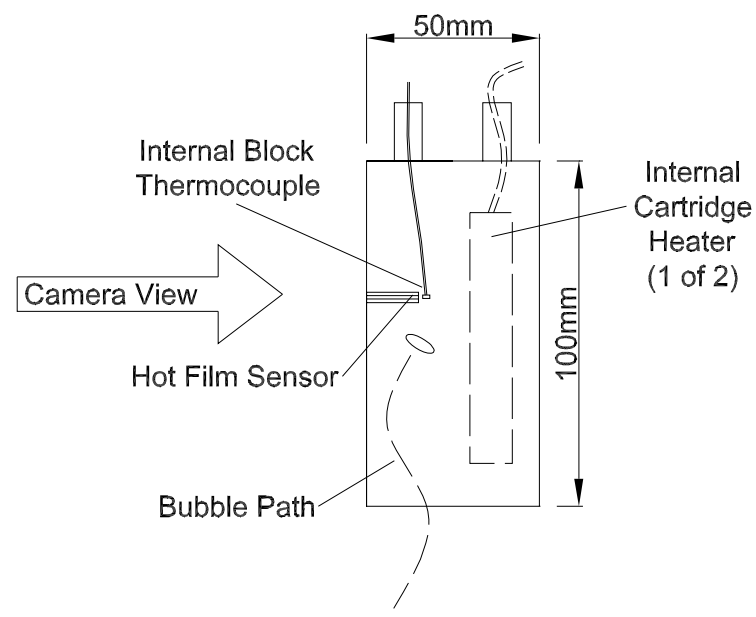

(b) Heated Test Surface

Figure 1: Schematic of Experimental Rig

The water is contained within a test tank that is made from $10 \mathrm{~mm}$ thick plate glass, $780 \mathrm{~mm}$ high with a square base of side $230 \mathrm{~mm}$. These dimensions were selected to provide sufficient water volume for the bulk water temperature to remain substantially constant throughout tests. The bubble injection system consists of a syringe (machined to remove the tip) mounted at the base of the tank on a movable platform. Air is fed to the syringe via a rubber hose connected to a plunger. A PC controlled servo motor presses the plunger thus supplying air to the syringe providing a single bubble of repeatable size.

Mounted within the glass tank is a copper block $100 \mathrm{~mm}$ high $\times 50 \mathrm{~mm} \times 30 \mathrm{~mm}$ (figure 1 (b)). The base of the block is $450 \mathrm{~mm}$ from the bubble injection point. Contained within the copper 
block are two $535 \mathrm{~W}$ cartridge heaters powered by a variable power source. The heated block approximates a uniform wall temperature boundary condition. Heat flux measurement is achieved using a Senflex model SF0105 hot film sensor manufactured by Tao Systems. It is flush mounted to the block $62 \mathrm{~mm}$ from the base and $10 \mathrm{~mm}$ from the centre line. The sensor is mounted horizontally i.e. perpendicular to the main flow direction. It consists of a very thin layer of nickel deposited on an electrically insulating substrate required for under water applications. The nickel layer is at maximum $0.2 \mu \mathrm{m}$ thick and is positioned between two $50 \mu m$ thick polyimide layers. The nickel sensing element is approximately $0.1 \mathrm{~mm}$ wide by $1.4 \mathrm{~mm}$ long. This results in a cold resistance of approximately $14 \Omega$.

The hot film sensor works in conjunction with a TSI (model 1053B) constant temperature anemometer. As the CTA passes current through the film it heats ohmically and heat is transferred from the film to the surroundings. The surface heat transfer rate between the sensor and the fluid flow is related to the amount of power required to maintain the film at a set temperature. The output voltage, $V$, of the bridge is monitored by the data acquisition system. Equations 1 to 4 below relate the heat dissipated to resistance, voltage and current.

$$
q_{\text {diss }}=R_{\text {film }} \times I^{2}
$$

where

$$
I=\frac{V}{R_{\text {top }}+R_{\text {probe }}}
$$

and

$$
R_{\text {probe }}=R_{\text {film }}+R_{\text {cable }}
$$

therefore

$$
q_{\text {diss }}=\frac{R_{\text {film }}}{\left(R_{\text {top }}+R_{\text {probe }}\right)^{2}} \times V^{2}
$$


The hot film is maintained at a temperature of $15^{\circ} \mathrm{C}$ above that of the heated block; this is required to give a significant output from the film, thus increasing the signal to noise ratio. A consequence of this is that some heat is dissipated to the block surface through conduction. In order to evaluate the heat convected to the fluid alone the film output voltage is first measured under zero convective flow conditions, i.e. when there is no temperature difference between the block and the water. Under this zero flow condition, the measured output voltage is a combination of conduction to the copper block, convection to the fluid due solely to the film overheat and radiation (negligible) to the surroundings. This acquired voltage $V_{0}$ is subsequently subtracted from the voltage measured during testing. Equation 4 now becomes:

$$
q_{\text {diss }}=\frac{R_{\text {film }}}{\left(R_{\text {top }}+R_{\text {probe }}\right)^{2}} \times\left(V^{2}-V_{0}^{2}\right)
$$

The geometric surface area of the hot film is given by the manufacturer to be $1.5 \times 10^{-7} \mathrm{~m}^{2}$. Due to conduction from the hot film to its surroundings the effective surface area has been shown by Beasley and Figliola [1988] to vary significantly from this. Heat generated by the film conducts into the copper block and in effect this increases the area over which heat is transferred from the film. In order to estimate the effective surface area comparisons must be made between the heat flux measured and theoretical calculations. Thus, the local heat transfer coefficient, $h$, for a heated vertical plate submerged in a fluid can be calculated from natural convection theory and used in conjunction with the measured temperature difference to calculate the effective surface area. This is indicated by equation 6:

$$
A_{\text {eff }}=\frac{q_{\text {diss }}}{h \Delta T}
$$

in which $q_{\text {diss }}$ is determined from equation 5 above. Natural convection tests at differing wall to bulk water temperatures were performed. The voltage through the hot film sensor was recorded at each setting along with the temperature difference and using equation 6 the 
effective surface area for each test was found to be a factor of 5.4 times that of the geometric surface area, which is consistent with the study by Beasley and Figliola [1988].

A T-type thermocouple is mounted just beneath the surface of the block at the level of the hot film sensor to measure the block temperature. A second thermocouple is suspended in the fluid away from the block in order to measure the bulk water temperature. It is maintained at the same vertical level as the other sensors. Data acquisition for the thermocouples and hot film sensor is carried out by a National Instruments data acquisition system composed of an NISCB-68 breakout board, with cold junction compensation, connected to an NI1036-E PCI card. This card works in conjunction with LabVIEW software, also supplied by National Instruments, used to set data acquisition frequency, display the output signals and record data. High speed photography is performed by a NAC HI-Dcam II digital camera and PCI board. Photos are acquired at 250 frames per second $(f p s)$. The camera is used in conjunction with HI-Dcam link v.3 software in order to apply camera settings and record data. Both LabVIEW and the digital camera are connected to a Thurly Thandar Instruments TGP 110, 10MHz pulse generator. Upon applying a trigger, this unit generates a pulse that starts synchronous data acquisition by the digital camera and the LabVIEW software. Data are acquired for 15 seconds at $5000 \mathrm{~Hz}$ for the thermocouple and heat flux sensors and the camera records at 250fps. Results are presented of the time resolved convective heat transfer coefficient which has a calculated uncertainty of $35.5 \%$. This takes account of both fluid and block temperatures but the main contributor to the uncertainty is the effective surface area.

\section{Results \& Discussion}

Synchronisation of the two data acquisition systems allows heat flux and bubble position to be analysed on an accurate time-line. The following sections assess bubble enhanced heat 
transfer, the effects of the orientation of the plane in which the bubble oscillates and the effects of bubble path proximity to the plate.

\subsection{Bubble Enhanced Heat Transfer}

Before the bubble is injected into the tank, heat is transferred by natural convection alone. This heat transfer coefficient is obtained from the hot film sensor and has been confirmed by theoretical calculations. Images of the bubble in motion are compiled by overlapping sequential images maintaining a constant time separation of $0.02 \mathrm{~s}$. These images aid in understanding the bubble motion and position of the bubble in relation to heat flux and temperature measurements. In addition, the bubble is seen to oscillate at a frequency of approximately $8 H z$ which is comparable with Saffman, [1956].

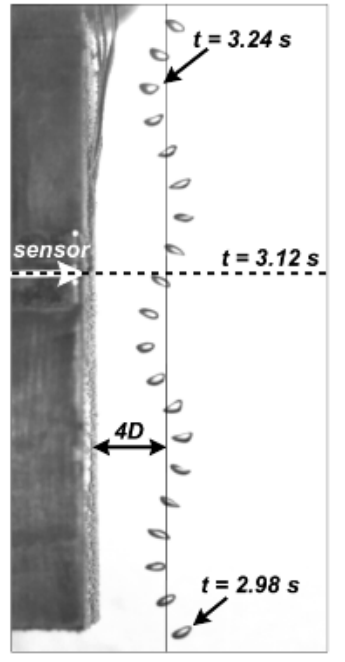

(a) Bubble Path

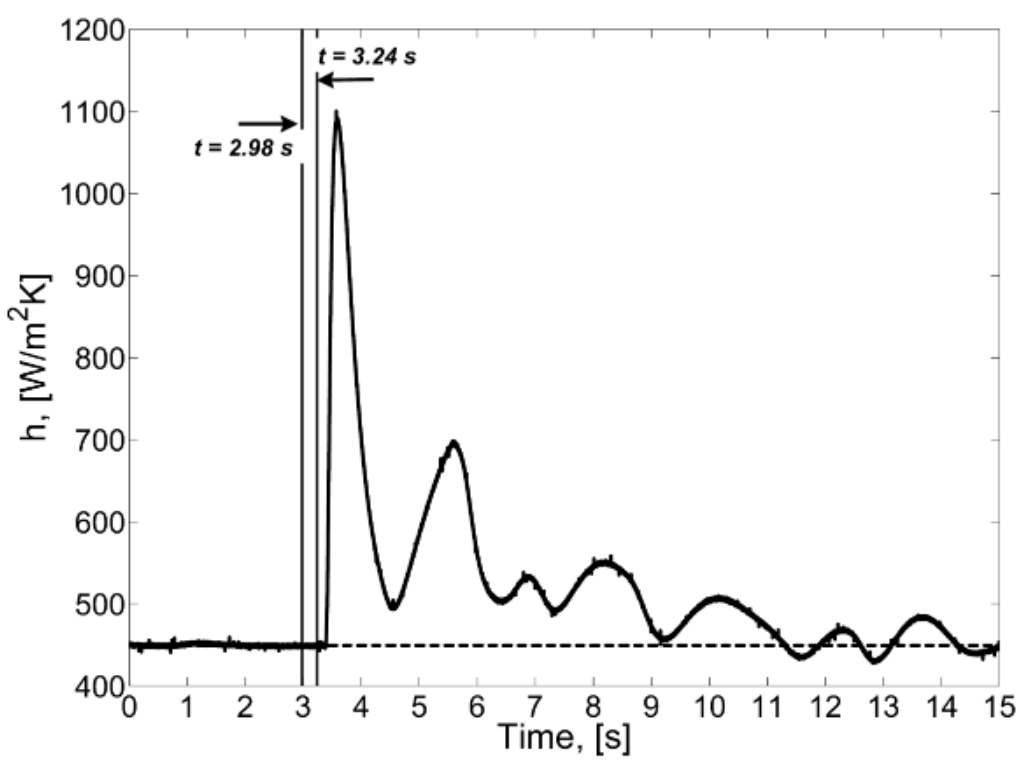

(b) Convective Heat Transfer Coefficient

Figure 2: Transient Evolution of Bubble Position and Surface Heat Transfer, $x / D=4$

The mean distance of the bubble path from the heated vertical surface is presented in terms of the bubble equivalent diameter $(x / D)$. Figure 2 (a) shows the heated test surface and the bubble location at regular time intervals for an elliptical bubble with major axis diameter of $4 m m$ passing at $x / D=4$. Figure 2 (b) presents equivalent measurements of the surface heat transfer coefficient. At $t=2.98 \mathrm{~s}$ from the start of data acquisition, the bubble is approaching 
the bottom of the block and the convective heat transfer coefficient is measured as $450 \mathrm{~W} / \mathrm{m}^{2} \mathrm{~K}$. The temperature difference between the bulk water and block is $15.9^{\circ} \mathrm{C}$ which yields a theoretical natural convection heat transfer coefficient very similar to the measured value, as indicated in figure 2 (b).

As can be seen from figure 2 (a), at $t=3.24 \mathrm{~s}$ the bubble is approximately $30 \mathrm{~mm}$ past the hot film sensor. The centroid of the bubble has a horizontal displacement of approximately $7 \mathrm{~mm}$ (i.e. the approximated sine wave has an amplitude of $7 \mathrm{~mm}$ ) and the minimum horizontal distance from the block to the bubble is approximately $10 \mathrm{~mm}$. Even though the bubble has passed the sensor the heat transfer level is unchanged at $t=3.24 \mathrm{~s}$. A delay exists between the time when the bubble is level with the hot film sensor $(t \approx 3.12 s)$ and when the enhancement effects are seen $(t=3.33 s)$. This delay is possibly the time it takes for the bubble wake to travel from the bubble to the heated surface and is discussed in a later section. At $t=3.33 \mathrm{~s}$ the wake impacts on the surface and a sharp peak in heat flux is seen in figure 2 (b). The increase can be seen to reach $1100 \mathrm{~W} / \mathrm{m}^{2} \mathrm{~K}$ at its peak which corresponds to 2.4 times the natural convection heat transfer coefficient; this is broadly consistent with the results presented by Delauré et al. [2003]. The magnitude of the surface heat transfer decreases sharply from the initial peak but the effects of the bubble are shown to last in excess of 12 seconds. Fluctuations in the surface heat transfer are most likely attributed to vortex shedding: as the bubble travels through the fluid it develops horseshoe vortices which shed at the inflection point as discussed by Saffman, [1956] and Brücker, [1999]. At $t=11.2 \mathrm{~s}$ and thereafter the heat flux fluctuates below natural convection levels, this is most likely a result of turbulent fluid motion carrying hot water over the hot film sensor, resulting in a lower local temperature difference and an instantaneous heat transfer rate less than that of natural convection. 


\subsection{Effects of Bubble Orientation}

When the bubble rises through the tank it oscillates in the characteristic pattern for bubbles of this size range, as described by Bhaga and Weber, [1981]. The plane in which it oscillates doesn't generally change as the bubble rises i.e. it remains at the same angle to the heated surface as documented by Ellingsen and Risso [2001], described as the principal plane of oscillation. It can be seen that the plane in which the bubble oscillates has a dramatic effect on heat transfer enhancement. Figure 3 (a) shows a bubble oscillating in a plane normal (at an angle of $90^{\circ}$ ) to the heated surface and figure 4 (a) shows a bubble oscillating in a plane parallel (i.e. $0^{\circ}$ ) to the surface.

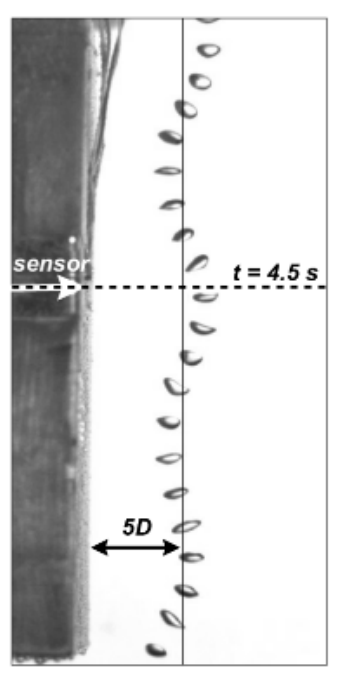

(a) Bubble Path

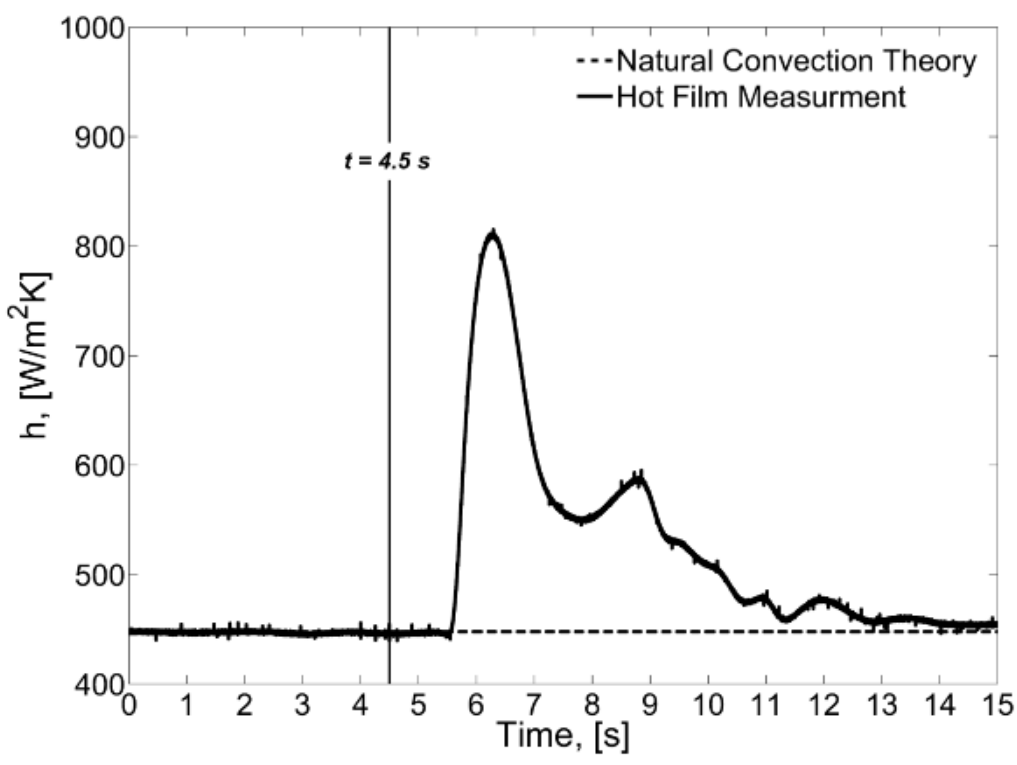

(b) Convective Heat Transfer Coefficient

Figure 3: Transient Evolution of Bubble Position and Surface Heat Transfer, $x / D=5,90^{\circ}$

It is clear from this that each bubble has a mean distance from the block of $5 D$ but each oscillates in a different manner. This has a major influence on the heat transfer coefficient as illustrated by comparison of figure 3 (b) and figure 4 (b). The vertical line illustrates when the bubble is at the level of the hot film sensor. 


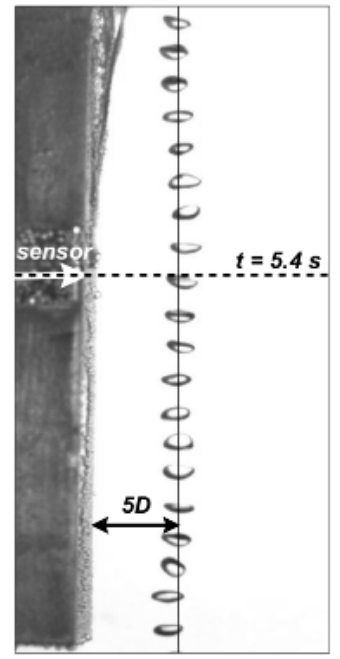

(a) Bubble Path

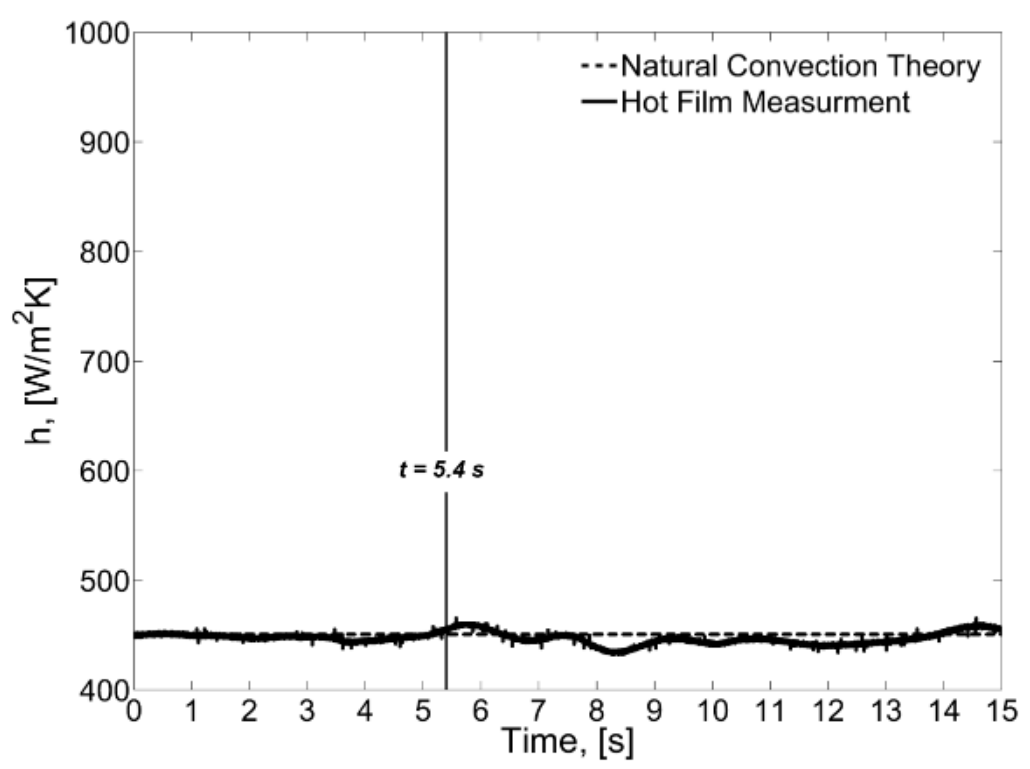

(b) Convective Heat Transfer Coefficient

Figure 4: Transient Evolution of Bubble Position and Surface Heat Transfer, $x / D=5,0^{\circ}$

A dramatic difference in the heat transfer can be seen between the two tests. This indicates that the mixing caused by the bubble has a direction associated with it. In the case where the bubble oscillates parallel to the block surface, the turbulent fluid motion appears to remain substantially within the same plane as it oscillates. The turbulent fluid motion never reaches the block where the sensor is located and therefore no increase in heat flux is seen. It is clear that in order to maximise the positive effects of the bubble on heat transfer it must oscillate in a plane perpendicular to the face of the heated block. Further research is still required in the field of flow visualisation and bubble imagery in order to better understand the fluid motion around the bubble and in its wake. It would be advantageous to simultaneously record the motion of the bubble from two angles orientated perpendicular to each other in order to obtain the position of the bubble in three dimensions. Understanding this is essential to understanding the mechanisms behind heat transfer enhancement.

It is important to note that only one high speed camera was available for this research and therefore only a two-dimensional representation of the bubble path can be presented. This 
makes it difficult to determine the bubble plane of oscillation relative to the block and it is also hard to establish whether the bubble passes directly in front of the hot film sensor. If the bubble plane of oscillation is not normal to the plate or if the bubble passes to the side, rather than in front of, the hot film sensor, it can lead to a lower enhancement effect recorded by the hot film sensor. The influence of the plane of oscillation has been addressed in this section while maintaining a constant mean distance from the plate for a similar sized bubble that passes directly in front of the hot film sensor.

\subsection{Effects of Bubble Proximity to Plate}

The distance from the bubble path to the block has a major influence on heat transfer. Images of the bubble path and heat transfer timelines are presented in this section for bubbles passing at distances of $6 D, 3 D$ and $1 D$ from the heated surface. The effects of bubble proximity are presented in figures 5 to 7 for tests in which, subject to the constraints of the experimental set-up, the bubble passes directly in front of the sensor and the plane of oscillation is normal to the surface.

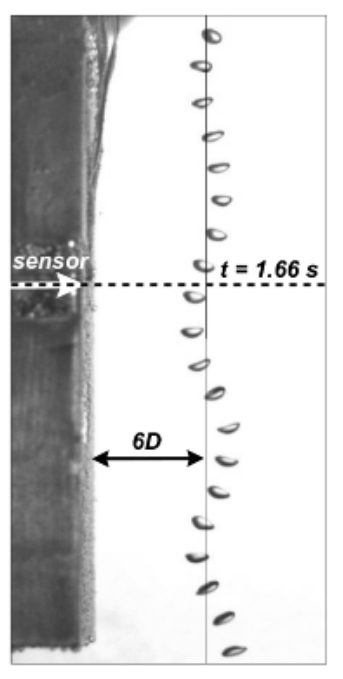

(a) Bubble Path

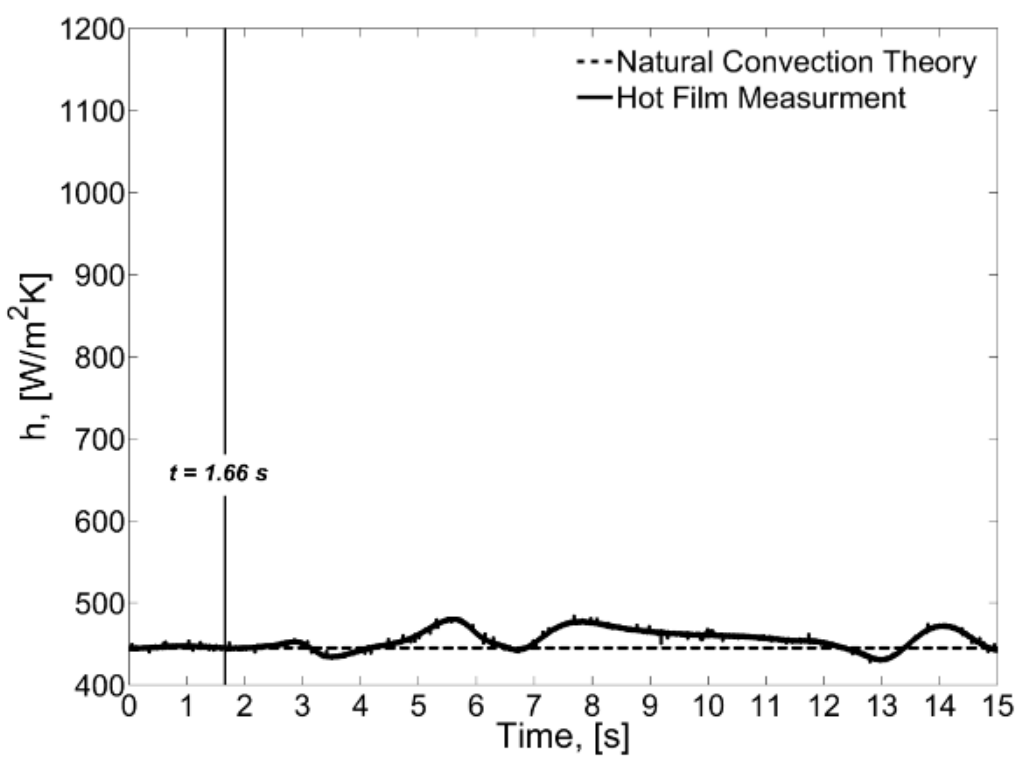

(b) Convective Heat Transfer Coefficient

Figure 5: Transient Evolution of Bubble Position and Surface Heat Transfer, $x / D=6$ 
Figures 5 (a) \& (b) show the bubble path and corresponding heat transfer for a mean distance of 6 bubble diameters. The vertical line in figure 5 (b) corresponds to the time when the bubble is level with the heat flux sensor. It can be seen from figure 5 (b) that the convective heat transfer coefficient is approximately $450 \mathrm{~W} / \mathrm{m}^{2} \mathrm{~K}$ before the bubble enters the test area (i.e. natural convection only) and no fluctuation in the heat flux can be seen until approximately 0.7 seconds after the bubble is level with the heat flux sensor. After this, heat flux levels elevate to a maximum value of $480 \mathrm{~W} / \mathrm{m}^{2} \mathrm{~K}$, which equates to an increase of approximately $7 \%$ over that of natural convection. Once again, heat transfer values are seen to drop below that of natural convection in certain areas of the test. This can possibly be attributed to the behaviour of the wake in drawing in warmer fluid. There exists a relatively large time delay between when the bubble is level with the sensor and when the first effects are seen (figure 5 (b)); this is due to the horizontal distance between the block and the bubble. Vortices created by the bubble have further to travel and therefore take longer to influence heat transfer at the surface. The heat flux enhancement is observed to be minimal. This may be due to viscous dissipation of the turbulent mixing created by the bubble; the elevated velocity is reduced by the damping effect of the surrounding water. 


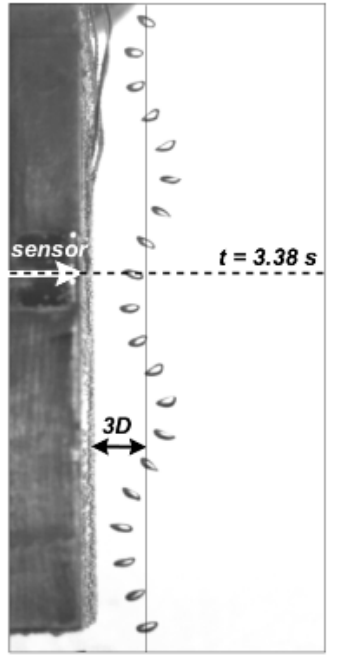

(a) Bubble Path

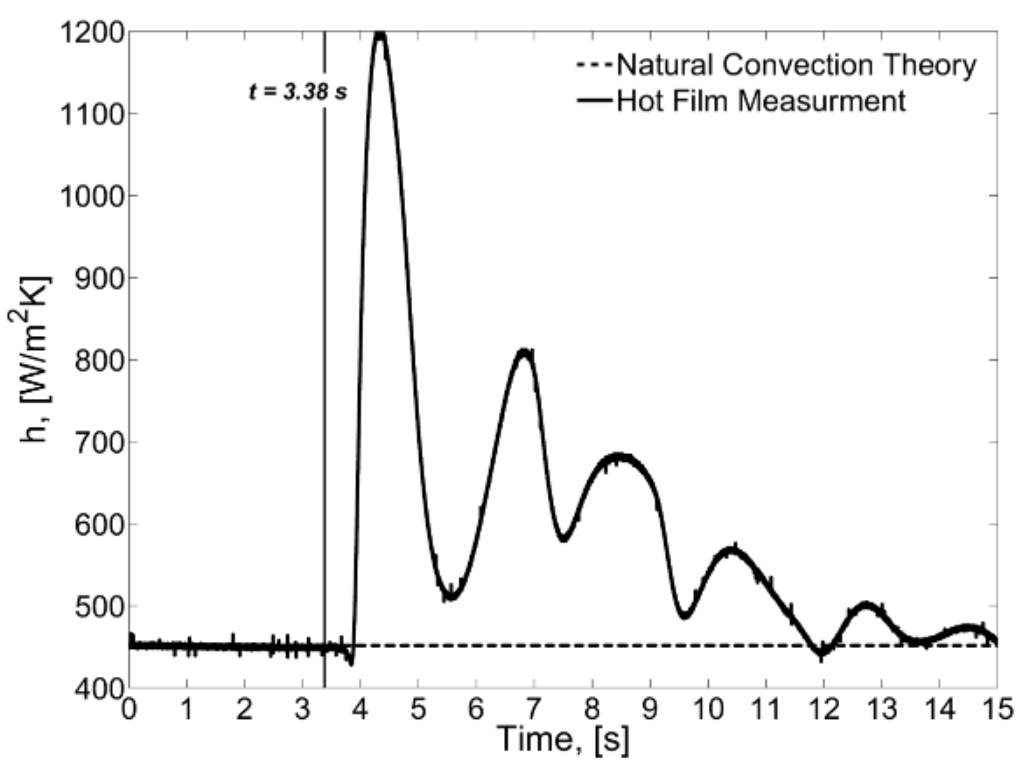

(b) Convective Heat Transfer Coefficient

Figure 6: Transient Evolution of Bubble Position and Surface Heat Transfer

When the bubble traverses a path closer to the block there is a significant change in both the time delay and the enhancement effects of the bubble. Results for a distance of $3 D$ are presented below in figures 6 (a) \& (b). The bubble passes the hot film sensor at $t=3.38 \mathrm{~s}$ and shortly afterwards $(\approx 0.2 s)$ the effects of this are seen. The temperature of the water surrounding the block decreases as the wake of the bubble impinges on the thermal boundary layer. This colder water is brought in from the surrounding bulk water and displaces the heated water. As a consequence of the colder water and the elevated fluid velocity, the heat flux increases from natural convection of $450 \mathrm{~W} / \mathrm{m}^{2} \mathrm{~K}$ to $1200 \mathrm{~W} / \mathrm{m}^{2} \mathrm{~K}$ and remains elevated (but fluctuating) for approximately the next 11 seconds. Due to the proximity of the bubble to the block, the wake now reaches the heated block quite rapidly after the passage of the bubble. Viscous damping effects are less significant also, resulting in significant increase in heat flux as compared with that at $6 D$ (figure 6 (b)). 


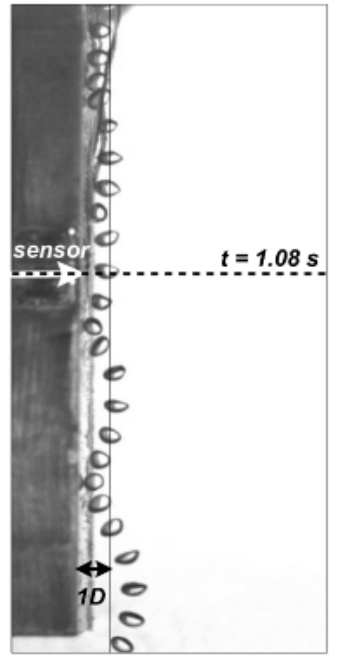

(a) Bubble Path

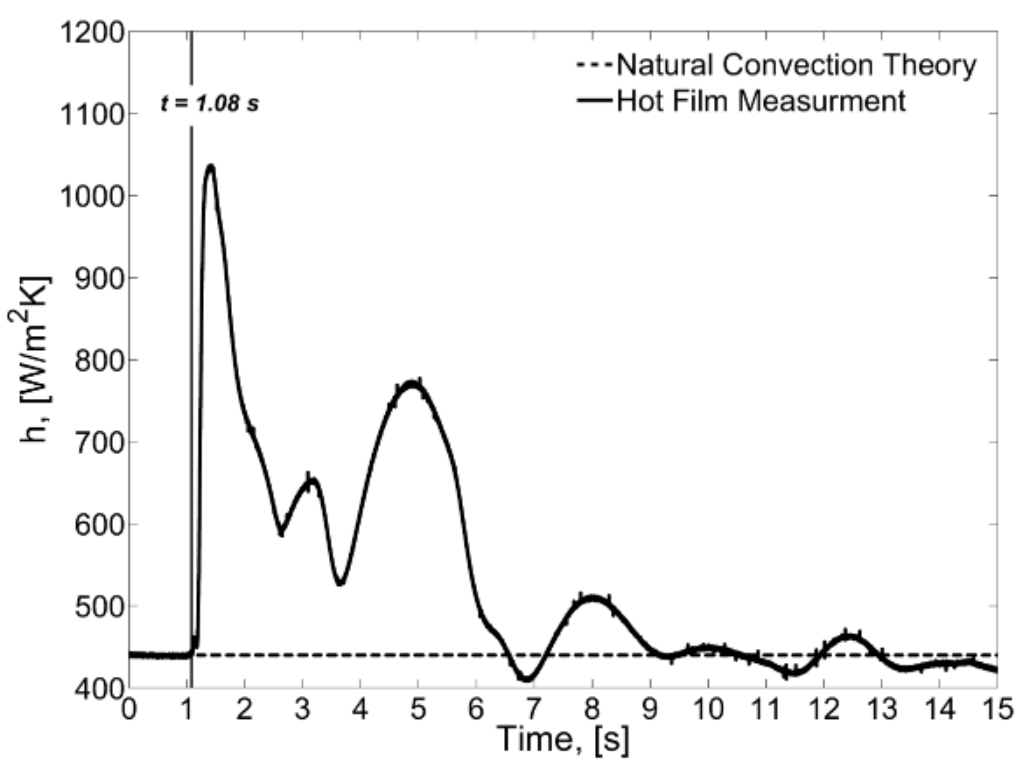

(b) Convective Heat Transfer Coefficient

Figure 7: Transient Evolution of Bubble Position and Surface Heat Transfer, $x / D=1$

Figure 7 (a) \& (b) illustrate the bubble path and transient evolution of heat transfer for a rising bubble path one bubble diameter from the heated surface. At a distance of $1 D$, the bubble can be seen to impact on the heated block and skim along its face. The bubble now acts like a bluff body disturbing the thermal boundary layer directly as it travels through it rather than relying on the indirect effect of the wake to increase heat flux (although the lasting effects are still attributed to the bubble wake). This explains why the effects on heat flux are seen approximately $0.03 \mathrm{~s}$ before the bubble reaches the level of the hot film sensor $(t$ $=1.08 \mathrm{~s}$ ). There is a dramatic increase in the heat flux, rising in a sharp peak from the natural convection level to $1040 \mathrm{~W} / \mathrm{m}^{2} \mathrm{~K}$ with effects lasting up to and beyond 14 seconds after the bubble is level with the sensor. The bubble appears to be drawn towards the block as it moves from bottom to top. This may be linked to the density gradient across the thermal boundary layer, or to the lower resistance offered by the natural convection flow close to the surface. The maximum heat flux observed in this case is slightly lower than that seen in the $3 D$ case $\left(1200 \mathrm{~W} / \mathrm{m}^{2} \mathrm{~K}\right)$ and although this is within the error limits it could also be due to the different 
mechanism by which the bubble disturbs the thermal boundary layer (i.e. the bubble itself disturbs the thermal plume rather than the bubble wake).

\section{Conclusions}

This paper reports on an experimental investigation of the dynamics of a rising bubble and its influence on heat transfer from a vertical heated plate. Heat flux measurements taken using a hot film sensor have shown that the rising bubble and its wake can have a significant effect on heat transfer. When the bubble rises close to the plate (within approximately $5 \mathrm{~mm}$ ) it acts like a bluff body, displacing the heated fluid, increasing local mixing and allowing cooler water to move in. For bubbles that rise further from the plate, it is the wake of the bubble that enhances heat transfer, the level of which is dependent on two parameters. Firstly, the closer the bubble is, the higher the increase in heat flux. Secondly, the orientation of the plane in which the bubble oscillates has a dramatic effect on enhancement. It is observed that when the bubble oscillates in a plane parallel to the heated plate, little positive effect on heat flux is seen, whereas, for the same sized bubble at a similar mean distance but oscillating in a plane perpendicular to the block face, a dramatic increase in heat flux is observed. It is also observed that a time delay exists between when the bubble passes the block and when the wake impacts; this is related to the displacement between the bubble path and plate. Further study is required in the field of flow visualisation in order to fully understand the phenomenon that occurs here.

\section{Acknowledgment}

This work was part supported by the Irish Research Council for Science, Engineering and Technology under grant SC/2004/E0077. 


\section{Nomenclature}

Symbol

$A_{\text {eff }}$

$D$

V

$h$

I

$q$

$R$

$t$

$T$

$x$
Description

Effective surface area of sensor

Equivalent bubble diameter

Voltage

Convective heat transfer coefficient

Current

Rate of heat transfer

Resistance

Time

Temperature

Perpendicular distance from surface
Units

$\left[m^{2}\right]$

[m]

[V]

$\left[\mathrm{W} / \mathrm{m}^{2} \mathrm{~K}\right]$

[A]

$[W]$

[Q]

$[s]$

$[K]$

[m] 


\section{References}

Atmane, M.A. and Murray, D.B., (2001) Bubble dynamics in nucleate boiling around a cylinder, Experimental Heat Transfer, Fluid Mechanics, and Thermodynamics, Pisa, Italy, pp. 1343-1348.

Beasley, D.E. and Figliola, R.S., (1988) A generalised analysis of a local heat flux probe, Journal of Physics E: Scientific Instrumentation, Vol. 21, pp. 316-322.

Bhaga, D. and Weber, M.E., (1981) Bubbles in viscous liquids: shapes, wakes and velocities, Journal of Fluid Mechanics, Vol. 105, pp. 61-85.

Brücker, C., (1999) Structure and dynamics of the wake of bubbles and its relevance for bubble interaction, Physics of Fluids, Vol. 11, pp. 1781-1796.

Cornwell, K. and Grant, I.A., (1998) Heat transfer to bubbles under a horizontal tube, International Journal of Heat and Mass Transfer, Vol. 41, pp. 1189-1197.

Delauré, Y.M.C., Chan, V.S.S. and Murray, D.B., (2003) A simultaneous PIV and heat transfer study of bubble interaction with free convection flow, Experimental Thermal and Fluid Science, Vol. 27, pp. 911-926.

Ellingsen, K. and Risso, F., (2001) On the rise of an ellipsoidal bubble in water: oscillatory paths and liquid-induced velocity, Journal of Fluid Mechanics, Vol. 440, pp. 235-268.

Manickam, S. and Dhir, V., (2003) Holographic interferometric study of heat transfer associated with a single vapor bubble sliding along a downward-facing heater surface, ASME Summer Heat Transfer Conference, Las Vegas, Nevada.

Qiu, D. and Dhir, V.K., (2002) Experimental study of flow pattern and heat transfer associated with a bubble sliding on downward facing inclined surfaces, Experimental Thermal and Fluid Science, Vol. 26, pp. 605-616.

Saffman, P.G., (1956) On the rise of small air bubbles in water, Journal of Fluid Mechanics, Vol. 1, pp. 249-275. 
Thorncroft, G.E. and Klausner, J.F., (1999) The influence of vapour bubble sliding on forced convection boiling heat transfer, ASME Journal of Heat Transfer, Vol. 121, pp. 73-79.

Yan, Y., Kenning, D. and Cornwell, K., (1997) Sliding and sticking vapour bubbles under inclined plane and curved surfaces, International Journal of Refrigeration, Vol. 20, pp. 583-591. 